\section{El desigual impacto de las políticas ferroviarias en una región transfronteriza. El caso de la Eurorregión Galicia-Norte de Portugal}

Mateo Varela Cornado

Facultad de Geografía e Historia, Universidad de Santiago de Compostela, España.

Recibido: 25 de septiembre de 2017. Aceptado: 14 de noviembre de 2017.

\begin{abstract}
Resumen
Galicia y el Norte de Portugal forman un espacio común dentro de la Península Ibérica. A pesar de pertenecer a dos estados diferentes, son muchas las similitudes entre estas dos regiones, como las culturales, paisajísticas o lingüísticas, donde la frontera política semeja no existir. La entrada tanto de España como Portugal en la antigua CEE (Comunidad Económica Europea) en 1986 y consigo la teórica libre circulación de mercancías y personas, supuso un aumento considerable de las relaciones internas. Esta última cuestión se vio reforzada por la construcción de nuevas infraestructuras, en especial, viarias, las cuales facilitaron los desplazamientos al verse reducido considerablemente los tiempos de viaje. A pesar del aumento de las relaciones internas desde hace 30 años y los altos niveles de movilidad transfronteriza, sigue existiendo un gran déficit en la oferta de transporte colectivo, en especial del ferrocarril. Aun de las grandes sinergias internas, las diferentes políticas ferroviarias de Portugal y España durante las últimas décadas tuvieron un impacto desigual en el territorio y con escasa incidencia para la integración del actual espacio que hoy forma la Eurorregión.
\end{abstract}

\section{The unequal impact of railway policies in a cross-border region. The case of the Euroregion Galicia-Norte de Portugal}

\begin{abstract}
Galicia and the North of Portugal form a common space inside the Iberian Peninsula. In spite of belonging to two different states, there are many similarities between these two regions, such as cultural, landscape or linguistic, where the political border seems not to exist. The entry of both Spain and Portugal into the old EEC (European Economic Community) in 1986 and with the theoretical free movement of goods and persons, marked a considerable increase in internal relations. This last issue was reinforced by the construction of new infrastructures, especially roadways, which facilitated the displacement of travel times. Despite the increase in internal relations for 30 years
\end{abstract}

Palabras clave

Galicia

Portugal

Ferrocarril

Movilidad

Eurorregión

Palavras-chave

Galiza

Portugal

Comboio

Mobilidade

Eurorregião

Keywords

Galicia Portugal Railway

Mobility

Euroregion 
and high levels of cross-border mobility, there is still a large deficit in the supply of public transport, especially rail. Despite the great internal synergies, the different rail policies of Portugal and Spain during the last decades had an unequal impact on the territory and with little incidence for the integration of the present space that today forms the Euroregion.

\section{Introducción}

Galicia y el Norte de Portugal forman un espacio singular dentro de la Península Ibérica (Palmeiro, 2011). El hecho de pertenecer a dos estados diferentes, España y Portugal, y la existencia de una frontera política, que semeja diluirse en los últimos años, no supone un impedimento para las fuertes relaciones entre los dos espacios. Son muchas las semejanzas entre estos dos territorios, culturales, paisajísticas o incluso lingüísticas. Además de la historia, tenemos que tener en cuenta que para Galicia siempre existió una mayor facilidad para las comunicaciones con su vecino del sur que con las demás regiones del estado español. Esto vino dado por la barrera física de las Sierras Orientales y las políticas de infraestructuras establecidas desde Madrid, las que mantuvieron a Galicia bastante alejada del resto de España hasta la década de los 1990s (Lois, Santos y Valcárcel, 2002). No sólo los factores geográficos y culturales provocaron esta fuerte relación. Más importante fue la entrada en la antigua CEE (Comunidad Económica Europea) por parte de Portugal y España en 1986, y consigo la teórica libre circulación de mercancías y personas, provocando un aumento considerable de los intercambios sociales y económicos. De hecho, el comercio exterior entre ambos espacios no ha dejado de crecer, existiendo una complementariedad entre las dos economías (Fdez y Grela, 2003; Trillo y Lois, 2011).

La entrada en el espacio europeo, trajo consigo nuevos organismos para la cooperación transfronteriza a diferentes escalas; locales, supralocales y regionales. Uno de estos nuevos organismos, las denominadas AECT (Asociación Europea de Cooperación Transfronteriza), dio origen a la Eurorregión Galicia-Norte de Portugal, formada por la Comunidad Autónoma de Galicia y la Región Norte de Portugal. Esta última no es una unidad administrativa como su vecina del norte, sino que se trata de una unidad estadística europea (NUTS) que aglutina a los distritos de Braga, Bragança, Porto, Viana do Castelo, Vila Real y ciertos concelhos (ayuntamientos en portugués) de los distritos de Aveiro, Viseu y Guarda (Figura 1).

Para que se dieran estos altos índices de intercambios, también son necesarias las infraestructuras de transporte para canalizar este aumento de movilidad. Durante las últimas décadas, se llevaron a cabo diferentes actuaciones, como la construcción de nuevos viaductos que salvan el río Miño, entre las localidades de Tomiño y Vila Nova de Cerveira o Salvaterra y Monção. También el acondicionamiento de nuevos pasos fronterizos mediante autopistas. El más reciente, entre Verín y Chaves, en el interior de la Eurorregión. Los numerosos intercambios comerciales y la alta movilidad transfronteriza entre Galicia y Portugal se ven mejor reflejados en el nuevo puente internacional entre Tui y Valença do Minho. De todos los puntos fronterizos entre Portugal y España, es este último el que cuenta con la IMD (Intensidad Media Diaria) en número de vehículos más alto (VVAA, 2017). Estas nuevas autopistas permiten una rápida movilidad, siendo posible realizar en aproximadamente 3 horas, el desplazamiento entre los dos puntos urbanos extremos de la Eurorregión, Porto y Ferrol.

A pesar de la construcción de numerosos kilómetros de autopistas a ambos lados de la frontera durante las últimas décadas (Pazos, 2011; Pacheco, 2001) sigue existiendo un enorme déficit en las conexiones transfronterizas mediante transporte colectivo 


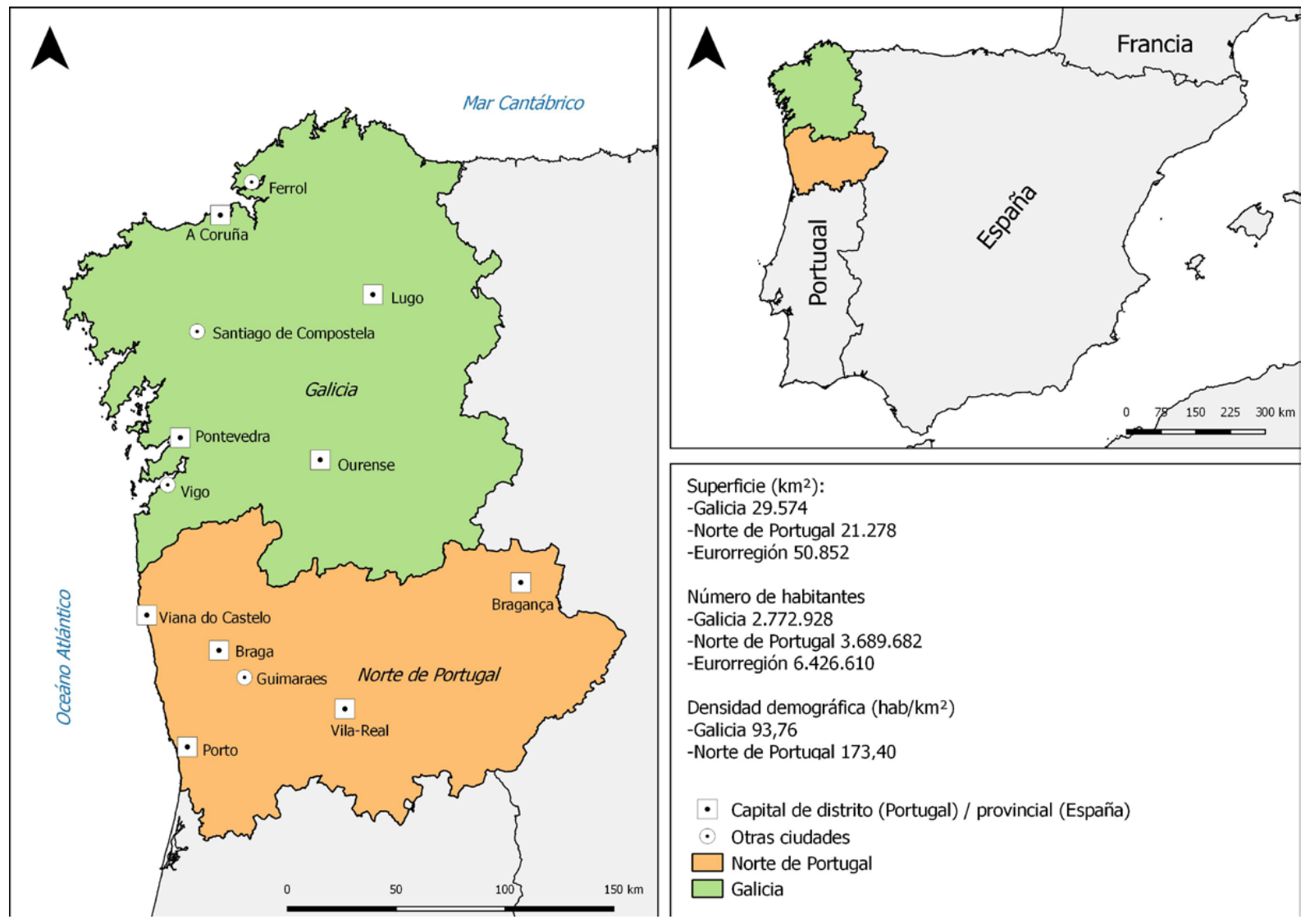

(Carballo, 2014) especialmente del ferrocarril. Donde tan solo existe una única línea internacional entre los dos espacios, por la cual transcurre el denominado desde 2013, el Tren Celta, entre Porto y Vigo. Este único servicio ferroviario entre dos de las principales ciudades de la Eurorregión, es comercializado en conjunto por las dos operadoras ferroviarias, Comboios de Portugal (CP en adelante) y Red Nacional de los Ferrocarriles Españoles (RENFE en adelante). Antes de la última remodelación de servicios, se llegó a cuestionar por parte de la operadora portuguesa la viabilidad económica de este servicio, debido al número bajo de usuarios y el gran déficit económico en su explotación. La posibilidad de cierre llegó a ser propuesta hasta en dos ocasiones, en el 2005 y el 2011. Tenemos que tener en cuenta la importancia de este servicio ferroviario por diferentes motivos. Por una parte, la mayoría de los desplazamientos entre Galicia y Portugal se realizan mediante vehículo privado, no sólo se trata de su impacto ambiental, también una discriminación cara ciertos sectores de la población (menores de 18 años y personas de avanza edad). Una segunda cuestión, es la vertebración interna no sola de la propia Eurorregión, también de todo el Eje Atlántico. La mayor parte de la fachada occidental atlántica de la Península Ibérica, desde el norte de Galicia hasta la capital lusa, Lisboa, se caracteriza por ser un espacio urbanizado continúo y con altas densidades demográficas. Siendo este uno de los espacios más dinámicos en términos socio-económicos dentro del continente europeo (Florida, Gulden y Mellander, 2008).

Figura 1. La Eurorregión Galicia-Norte de Portugal. Fuente: Instituto Galego de Estadística 2017 (IGE) e Instituto Nacional de Estatística 2017 (INE).

\section{Metodología}

La metodología a llevar en la presente investigación será diversa. Por una parte, realizaremos una revisión bibliográfica de las principales obras escritas en los dos espacios para tratar de explicar la historia y posterior evolución de la red ferroviaria. También 
obras e investigaciones con todo lo relacionado con los efectos inducidos en el territorio y el espacio por el ferrocarril, para de esta manera tratar de aplicarlos a la realidad de la Eurorregión, como es el caso del tren de alta velocidad o los servicios de cercanías.

Por otra parte, también se utilizarán los diferentes documentos de planificación de infraestructuras de transporte a ambos lados de la frontera. Estos establecieron y marcarán el futuro de las diferentes líneas ferroviarias en los dos espacios. Al mismo tiempo, al referirnos a un espacio supra-estatal, tienen especial incidencia las diferentes políticas y directrices establecidas por la Unión Europea, como las ediciones del Libro Blanco de Transporte de los años 2001 y 2011 o el proyecto de la Red Transeuropea de Transporte del 2005. Con respecto a la infraestructura actual, para tener en cuenta sus características y denominaciones, utilizaremos aquellos documentos elaborados por las empresas públicas encargadas de la construcción y mantenimiento de las infraestructuras ferroviarias. En Galicia, ADIF (Administración e Infraestructuras Férreas) y por Portugal, Infraestruturas de Portugal.

No solo los documentos referidos a la planificación y desarrollo de infraestructuras, con respecto a la información cuantitativa sobre el número de usuarios y su evolución, utilizaremos aquellos datos subministrados por diferentes organismos públicos. Este es el caso de la movilidad entre Galicia y Portugal, por el Observatorio Transfronterizo España-Portugal. Sobre el número de usuarios del ferrocarril, tan sólo analizaremos aquellos que se encuentran disponibles al público en general, en el Observatorio del Ferrocarril de España y el Relatorio anual de la operadora portuguesa Comboios de Portugal. No obstante, las características de los servicios, es decir, número de frecuencias, tipología y horarios, en las propias páginas web de las dos operadores ferroviarias, RENFE y CP.

Otra cuestión será el análisis mediante cartografía temática, de este modo explicaremos de mejor manera ciertas cuestiones como la distribución de las diferentes líneas ferroviarias o la relación de estas con la ocupación del territorio por parte de la población. Para esta última tenemos que tener en cuenta las diferentes realidades con respecto a las unidades administrativas. Por esta razón, escogimos aquella unidad administrativa que más semejanza tiene en número y superficie media a ambos lados de la frontera. Por parte de Galicia, la parroquia y en el Norte de Portugal, la freguesia. Sobre la información demográfica, usaremos el año 2011, ya que se trata del último año en el que se realizó un censo de población en los dos espacios. Esta información es obtenida directamente de los dos organismos estadísticos, el Instituto Galego de Estadística (IGE en adelante) y el Instituto Nacional de Estadística (INE en adelante).

\section{Estado de la cuestión}

La gran dotación de infraestructuras de transportes es una de las principales características de las economías más punteras. Estas fueron y siguen siendo utilizadas en numerosas ocasiones, no sólo como instrumentos para la vertebración territorial, sino para el desarrollo territorial. Durante las dos últimas décadas hemos vivido, tanto en Galicia como en el Norte de Portugal, una auténtica expansión espacial de las infraestructuras por ambos espacios, desde las carreteras convencionales, autopistas, nuevas terminales aeroportuarias hasta la llegada del tren de alta velocidad. Bien es cierto que la propia dotación de infraestructuras de transportes no provoca de por si un mayor desarrollo territorial (Bel, 2010). Más allá de las consecuencias, negativas o positivas, en la economía de un estado o región, las infraestructuras y los diversos medios de transporte también tienen una serie de repercusiones territoriales, espaciales o sociales. En primer lugar, durante la construcción y antes de que entren en servicio, las propias 
infraestructuras ya provocan una serie de alteraciones en el territorio y el paisaje, con movimientos de tierra, nuevos viaductos o túneles (Bellet, 2013). Con un mayor impacto según el nivel de complejidad de las obras. Esto se puede comprobar fácilmente en Galicia, con la llegada de la alta velocidad ferroviaria, donde fue necesaria la construcción de numerosos viaductos, muchos con una longitud superior al kilómetro.

Centrándonos especialmente en el ferrocarril, debemos diferenciar los efectos espaciales inducidos por las diferentes tipologías de este modo de transporte. No es el mismo efecto corredor del tren regional o metropolitano, donde la expansión de los beneficios es continuo en la distancia y con gran accesibilidad (numerosas estaciones) a lo largo de la línea ferroviaria (Gutiérrez, 2004) que el efecto túnel del tren de alta velocidad, donde el espacio se configura de manera descontinua, siendo este último modelo ferroviario más semejante al avión que al tren convencional (Troin, 1995). Además, otras consecuencias como el reforzamiento de los núcleos de población que dispongan de una estación accesible a la nueva red, modificaciones en el sector turístico con el aumento del excursionismo o el aumento de la movilidad debido a las mayores facilidades y la mejora de los tiempos de desplazamiento entre las ciudades.

La alta movilidad es una de las principales características de las sociedades occidentales (Urry, 1999). Las sociedades tanto de Galicia como del Norte de Portugal también comparten esta característica con una gran dependencia del vehículo privado, de ahí la expansión de la nueva red viaria (Pazos, 2003; Pacheco, 2001). Este aumento de la movilidad, no solo se dio internamente en cada uno de los dos espacios, también entre ellos, es decir, trans-fronteriza. Palmeiro y Pazos (2008) y Carballo (2014) tienen analizado este aumento de la movilidad entre Galicia y Portugal, pero con graves problemas en materia de transporte colectivo, especialmente del ferrocarril a pesar de las diferentes directivas europeas para la integración de los sistemas ferroviarios. Una cuestión importante que superaría los problemas actuales en la gestión compartida de los servicios internacionales.

Por último, Fernández (2006) y Rodríguez y Pérez (2007) describen la evolución del ferrocarril y la falta de conexiones entre los dos espacios, dificultando la vertebración territorial de la Eurorregión.

\section{Período antes de la entrada a la CEE}

Pero para comprender la escasa incidencia del ferrocarril en la vertebración interna de la Eurorregión Galicia-Norte de Portugal, es necesario realizar un pequeño análisis sobre la evolución de las diferentes líneas ferroviarias en los dos espacios desde sus inicios. Hasta la llegada de los nuevos servicios de altas prestaciones y alta velocidad a comienzos del siglo XXI, la mayor parte de los trazados de las vías férreas, tanto de Galicia como del Norte de Portugal, databan de finales del siglo XIX y principios del XX. En la actualidad, ciertos tramos ferroviarios siguen teniendo el mismo trazado que el día de su inauguración, muchos de ellos, con más de 100 años de antigüedad (Fernández, 2006). Tenemos que tener en cuenta que se trata de un momento alejado del actual marco contemporáneo de cooperación transfronteriza, donde primaban cuestiones diferentes a las de hoy en día.

La instalación del ferrocarril al espacio que hoy forman Galicia y el Norte de Portugal llegó con cierto retraso con respecto a otros territorios próximos. La primera línea que se inauguró en la Península Ibérica, concretamente en Cataluña, fue la Barcelona-Mataró en 1848. Mientras que ocho años después, era abierto al servicio el primero tramo ferroviario en el estado luso, entre Lisboa y Carregado, en 1856. 
El tren no llegaría al Norte de Portugal hasta 1864, año en el que la Linha do Norte finalizaba en Vila Nova de Gaia, ayuntamiento localizado al sur de Porto. Tuvieron que transcurrir 12 años, para que finalmente el ferrocarril llegase hasta la segunda ciudad lusa, luego de que se inaugurase el Puente de María Pía para cruzar el río Duero (Alegria, 1990). Por otra parte, en Galicia, la primera línea ferroviaria que se puso en marcha fue en 1873, entre Carril (Vilagarcía de Arousa) y Cornes (Santiago de Compostela). Esta se puede considerar como la única línea férrea planificada desde un punto de vista gallego, ya que el principal objetivo era la conexión de la actual capital gallega con un puerto (Fernández, 2006). Tenemos que tener en cuenta que la planificación férrea en España estará marcada por la "Ley General de Caminos de Hierro" de 1855. Con esta ley se instauraba una red radial con centro en Madrid, aún presente en la actualidad. Esto provocó una escasa incidencia de este medio de transporte en España (Bel, 2010). Por lo tanto, la prioridad de las líneas gallegas era la conexión con la Meseta y no la búsqueda de una vertebración territorial interna. Esto se pudo comprobar en la falta de una línea ferroviaria directa entre las dos principales ciudades gallegas, A Coruña y Vigo, que data de 1943, cuando a finales del siglo XIX, ya disponían de conexión con Madrid mediante la única salida, vía Monforte de Lemos y Ponferrada.

Así como el objetivo en la planificación férrea en Galicia era conseguir conexiones con la Meseta y Madrid, las primeras líneas portuguesas, además de tener como objetivo ligar los principales núcleos de población y los puertos marítimos, procuraban llegar hasta la frontera terrestre con España para poder aumentar las relaciones comerciales con el exterior (Alegria, 1990). De ahí que entre Galicia y Portugal se establece en 1886, el único paso fronterizo mediante ferrocarril, vigente en la actualidad, con la inauguración del Puente Internacional de Tui-Valença do Minho. Al igual que en Galicia, durante las últimas décadas del siglo XIX, se construyen diversas líneas ferroviarias en el norte de Portugal, como la Linha do Minho o la Linha do Douro. Estas dos se marcaban dentro de las prioridades marcadas desde las autoridades portuguesas para finalizar en la frontera con España.

El cambio de siglo, no supone grandes consecuencias en materia ferroviaria en Galicia. Ya que tan sólo se completa la llegada del ferrocarril a la ciudad de Ferrol en 1913 y posteriormente al conflicto bélico de la Guerra Civil (1936-1939), el tramo entre A Coruña y Santiago de Compostela en 1943 y la salida por el sur entre Ourense y Zamora. Para esta última existían proyectos diferentes al establecido finalmente. Una de las variantes recogía la dotación de un segundo punto de paso ferroviario entre Galicia y el Norte de Portugal, mediante las localidades de Verín y Chaves (Blanco, 2014). Por otra parte, en el Norte de Portugal, se concluyen un número destacado de líneas ferroviarias; Vouga, Guimarães, Tâmega, Corgo, Tua y Sabor. Excepto las dos primeras, las cuatro restantes salen de la Linha do Douro cara el norte siguiendo el trazado de los ríos. Al igual que en Galicia, donde el relevo tuvo un gran peso a la hora de escoger los diferentes trazados de las líneas ferroviarias (Nárdiz, 1996), los valles acentuados en el Norte de Portugal determinaban las dificultades para las conexiones directas transversales entre el interior y el litoral portugués. Por lo que la mejor manera de vertebrar todo este espacio era con el establecimiento de las diferentes vías férreas a lo largo de los diversos valles fluviales y posterior enlace con la vía principal, en este caso, la Linha do Douro (Rodríguez y Pérez, 2007). Tenemos que destacar además que estas líneas, disponían todas de ancho métrico $(1000 \mathrm{~mm})$. Una diferencia con respecto a Galicia, donde tan sólo se estableció una única vía férrea con esta tipología de ancho, entre Ferrol y Ribadeo, inaugurada en 1969. Además, durante estas primeras décadas del siglo XX, existe un cambio de tendencia ferroviaria en la planificación ferroviaria de Portugal. Los nexos con España ya no eran la prioridad, si no, una mayor vertebración territorial interna, de ahí la finalización de numerosas líneas ferroviarias (Alegria, 1990). 
Hasta la entrada de los dos países en la CEE en 1986 y la llegada de alta velocidad en el siglo XXI, tan sólo se puede destacar pequeñas actuaciones a ambos lados de la frontera como la electrificación. Una cuestión que en el Norte de Portugal llegó en 1966 para la conexión entre Porto y Lisboa, mientras que en Galicia tan sólo se dotó de esta tecnología a la línea entre Vigo y Ponferrada en 1982.

\section{La entrada en la CEE y posterior evolución}

La llegada de las democracias a Portugal en 1974 y España en 1977, abrió un nuevo período socio-económico en los dos estados, además de la posterior entrada en la antigua CEE en 1986. La universalización del vehículo privado permitió mejorar la accesibilidad de las personas, pero también fue la construcción de nuevas carreteras, las que permitieron recortar considerablemente los tiempos de desplazamiento. Las administraciones públicas de los dos espacios tuvieron durante las últimas décadas del siglo XX, una gran preocupación por la dotación de carreteras de altas capacidades. Es el caso de Galicia, que a partir de 1981 con la consecución de la autonomía, se fue construyendo una densa red de carreteras, desde las convencionales hasta autopistas (Pazos, Varela y Lois, 2015). Lo mismo acontecía en Portugal con los diversos PRN (Plano Rodoviario Nacional) de 1985 y 2000 (Pacheco, 2001). Este hecho, unido a la falta de inversiones y a la nula planificación de los servicios comerciales (frecuencias y horarios), fueron las principales causas que provocaron una caída considerable del número de usuarios del ferrocarril. Lo que provocó una situación económicamente insostenible en las dos operadoras, Comboios de Portugal y RENFE. Ante esto, las consecuencias fueron muy diferentes a ambos lados de la frontera galaico-lusa.

Por una parte, en Galicia, el gobierno autonómico contribuyó al mantenimiento de ciertos servicios deficitarios mediante una subvención desde 1995. Esta aportación económica duró hasta el año 2011, aunque dos años después, en el 2013, se establecía el cierre definitivo de varios apeaderos y frecuencias mediante un nuevo paquete de recortes ferroviarios por parte del gobierno central de España. Bien es cierto que la mayoría de estas estaciones se encontraban en zonas rurales, alejadas de sus núcleos de población de referencia y con un número escaso de frecuencias diarias. En ciertos casos, tan sólo una diaria por sentido. A diferencia de otras CCAA del Estado Español, en Galicia, no se llevó a cabo una clausura de líneas. Los diferentes acuerdos, denominados Contrato Programa, entre RENFE y el gobierno central durante la década de los 1980s, procuraban reducir considerablemente el déficit de la operadora, de ahí el cierre de numerosos kilómetros de vías férreas por la geografía española (Ramos, 2002).

Por otra parte, en Portugal las consecuencias fueron más drásticas. Así como en Galicia hubo una pérdida de servicios y apeaderos, pero nunca se llegaron a cerrar completamente las líneas, sobre todo porque sirven de nexo entre las principales ciudades, tanto gallegas como españolas. En el norte de Portugal fueron varias las líneas que quedaron sin servicio, siendo levantadas las vías poco tiempo después. Muchos de estos tramos fueron reconvertidos en vías verdes. A finales de la década de los 1980s tan sólo se cerró completamente el tráfico en la Linha do Sabor, y ciertos tramos de las demás líneas, Corgo, Douro, Tâmega, Tua o los ramales de Fafe en la de Guimarães y el de Monção en la del Minho. Recientemente, desde el estallido de la crisis económica en el 2008, cerraban por completo las líneas de Tâmega y Corgo. En el caso de Tua, sigue en funcionamiento un pequeño tramo intermedio, el llamado Metropolitano de Mirandela. La gran mayoría de estas líneas fueron inauguradas en los principios del siglo XX, con grandes limitaciones en cuanto a características técnicas, lo que limitaba considerablemente las velocidades máximas de los servicios (Comboios de Portugal, 2017). Además, estas líneas ferroviarias remataban en núcleos de población no destacados en cuanto 


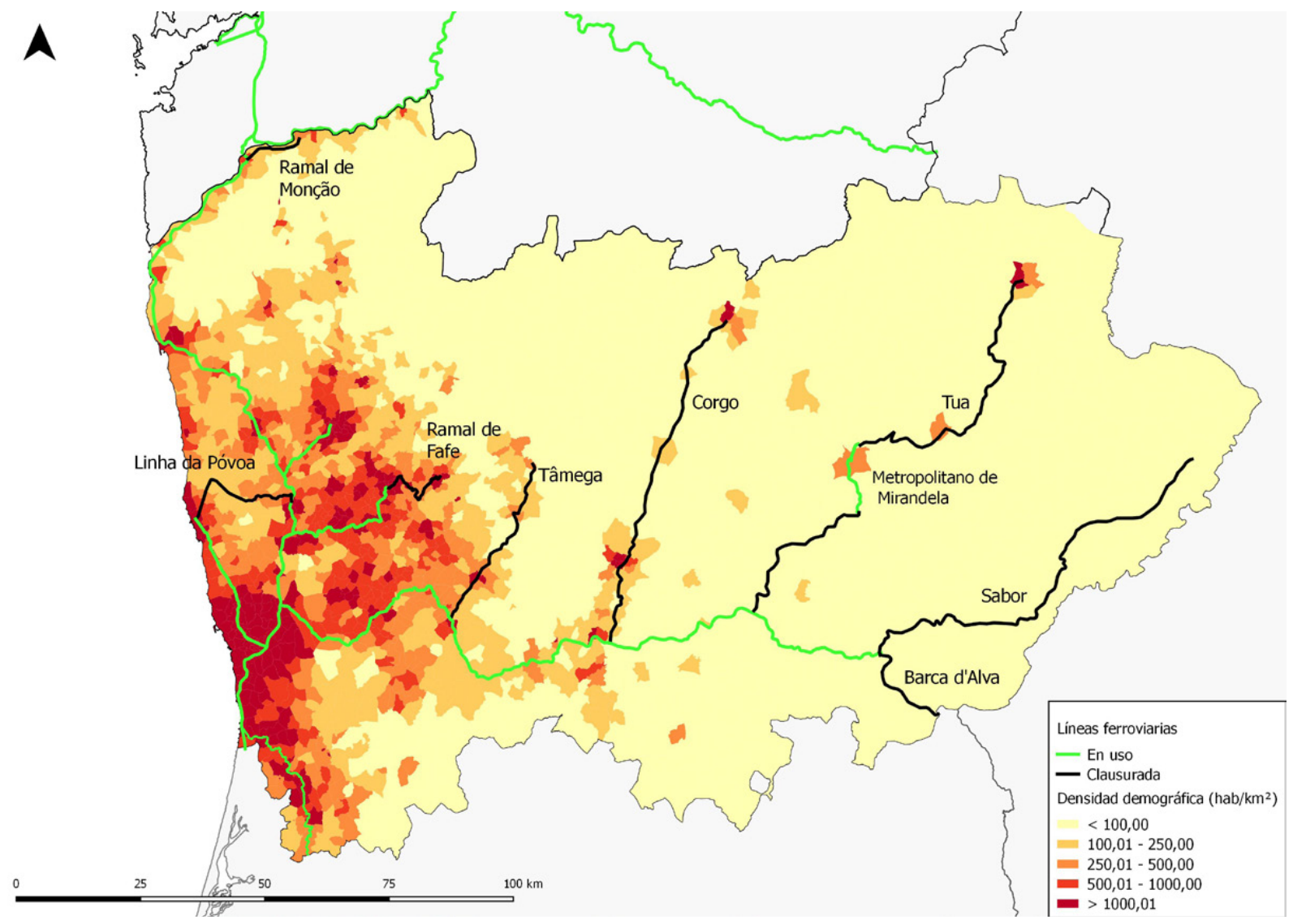

a número total de habitantes, como Bragança, Cabeceiras de Basto, Chaves, Miranda do Douro o Monção, áreas que a partir de la década de los 1950s iniciaron una fase de regresión demográfica (Figura 2). Tenemos que tener en cuenta que el ferrocarril como medio de transporte colectivo precisa de altas densidades de población, un hecho que no se produce en el interior de Portugal, al igual que en Galicia. Tampoco favorecía su explotación las características de la red ferroviaria, así como la malla gallega se encuentra totalmente cerrada y con salidas al exterior, las líneas del Norte de Portugal terminaban en estaciones de fondo de saco, lo que unido al bajo número de usuarios potenciales, provocaba que estas líneas estuvieran condenadas a un hipotético cierre.

Hay que tener presente el período de la construcción de la mayoría de las vías ferroviarias, tanto de Galicia como del Norte de Portugal, enmarcado en un momento donde el tren semejaba substitutivo del transporte por carretera (Nárdiz, 1996) de ahí la expansión de este medio de transporte, revolucionario en sus comienzos, sobre todo en los espacios del interior, donde existía una menor accesibilidad con respecto a los costeros (Gutiérrez, 1998).

A pesar de la pérdida continúa de protagonismo como medio de transporte desde mediados del siglo XX, sobre todo para los desplazamientos a media y larga distancia, el ferrocarril vuelve a cobrar importancia con la llegada del tren de alta velocidad en el continente europeo (Troin, 1995). En 1992, se inauguró la primera línea de alta velocidad en España, entre Madrid y Sevilla, pero fueron los diversos planos estratégicos de infraestructuras de los sucesivos gobiernos centrales, los que apostaron por este modelo de ferrocarril y su expansión por toda la geografía española. Esto provocó que España sea uno de los principales países punteros en dotación de líneas de alta velocidad ferroviaria (Albalate y Bel, 2011). Al igual que las líneas convencionales, la expansión de la alta velocidad siguió un modelo radial desde Madrid.
Figura 2. Líneas ferroviarias clausuradas en el Norte de Portugal y densidad de población en el 2011. Fuente: CP 2017 e INE 2017 (Instituto Nacional de Estadística). 
Por otra parte, en Portugal también se llevó a cabo un fuerte debate sobre la posibilidad de la dotación de este nuevo modelo ferroviario. Fueron varios los proyectos para la construcción de diferentes líneas de alta velocidad, las cuales se llegaron a presentar (Infraestruturas de Portugal, 2017). Al mismo tiempo, era creada una empresa pública, hoy absorbida por Infraestructuras de Portugal, para el estudio y gestión de estas futuras líneas, la RAVE, Rede Ferróviaria de Alta Velocidade SA. No sólo se planificó la dotación interna en el estado luso, cómo era la puesta en marcha de una nueva línea entre las dos principales ciudades, Porto y Lisboa, también 4 nuevas conexiones con España. Entre estas, una nueva línea a construir entre Porto y Vigo. El estallido de la crisis económica en el 2008, obligó a las autoridades portuguesas a abandonar estos proyectos, al contrario del modelo español. En la actualidad, uno de los principales esfuerzos económicos es para el nuevo acceso a Galicia, entre Ourense y Zamora. A pesar de no disponer del tren de alta velocidad, desde 1999 ya existe un servicio de altas prestaciones entre las principales ciudades de Portugal, el Alfa Pendular.

La entrada en la antigua CEE en 1986, tanto de Portugal como España, así como las diferentes directivas europeas, hay que tener en cuenta que dentro de la Eurorregión se sigue diferenciando la aplicación de dos políticas ferroviarias diferentes que no ayudaron a mejorar la vertebración interna, como veremos más adelante. De hecho, desde la entrada en el siglo XXI, se llevaron importantes actuaciones en las infraestructuras férreas que facilitaron la movilidad de los usuarios. Especialmente en Galicia con la nueva red de altas prestaciones, o en el Norte de Portugal, en el entorno del Gran Porto, pero no en el único servicio internacional entre las dos regiones. Es más, incluso podemos afirmar que hubo cierto retroceso. Las frecuencias diarias entre Vigo y Porto eran 3 (Díaz, 2002). No sólo la disminución de los servicios entre estas dos ciudades, antaño llegó a existir un servicio continúo en todo el Eje Atlántico, entre A Coruña y Lisboa (Fernández, 2006).

La expansión de las nuevas líneas ferroviarias en España llegó a Galicia en el 2001, con el inicio de sus obras, pero no fue hasta el 2009, cuando se inauguró el primer tramo de altas prestaciones entre dos ciudades gallegas, Santiago de Compostela y A Coruña, actualmente separadas por 30 minutos. En el 2011, entraba en servicio el primer tramo de alta velocidad (velocidades máximas superiores a los $300 \mathrm{~km} / \mathrm{h}$ ) en Galicia, entre Ourense y Santiago. A diferencia de las líneas de altas prestaciones de Galicia (el caso del Eje Atlántico), donde muchos de sus tramos tan sólo fueron reconvertidos para conseguir velocidades máximas superiores a los $200 \mathrm{~km} / \mathrm{h}$. La línea de alta velocidad, es totalmente nueva y de uso exclusivo para servicios comerciales de pasajeros. El último hito importante en Galicia, llegaba en el 2015, con la conexión directa entre Vigo y A Coruña, donde el desplazamiento es de tan sólo 70 minutos. En la mayoría de los casos, la reducción de los tiempos, es aproximadamente la mitad o incluso más de los establecidos antes de la llegada del nuevo tren.

La puesta en marcha de los nuevos servicios en Galicia tuvieron una fuerte repercusión en la movilidad y en el espacio (Pazos et al., 2015). Aun de no tratarse de servicios de alta velocidad, sobre todo en el caso del Eje Atlántico, los efectos espaciales inducidos por los nuevos trenes de altas prestaciones son muy semejantes a los de la alta velocidad de otros territorios, teniendo en cuenta que estamos analizando una escala más pequeña. Las consecuencias del tren de alta velocidad estudiadas y analizadas en otros territorios de España o Francia (Troin, 1995; Gutiérrez 2004; Bellet 2013), como la reducción de los tiempos de desplazamiento, nuevos proyectos urbanísticos en torno a las estaciones centrales, el aumento de las relaciones entre diferentes ciudades o posibles cambios en los hábitos de las personas gracias a frecuentes y rápidos servicios, se dan ya en Galicia. De hecho, los días de mayor afluencia, viernes y domingos, especialmente durante el curso escolar universitario, suele ser muy frecuente la falta de plazas libres en diferentes horarios. Esto obliga al potencial usuario a planificar con varios días de antelación su viaje o 


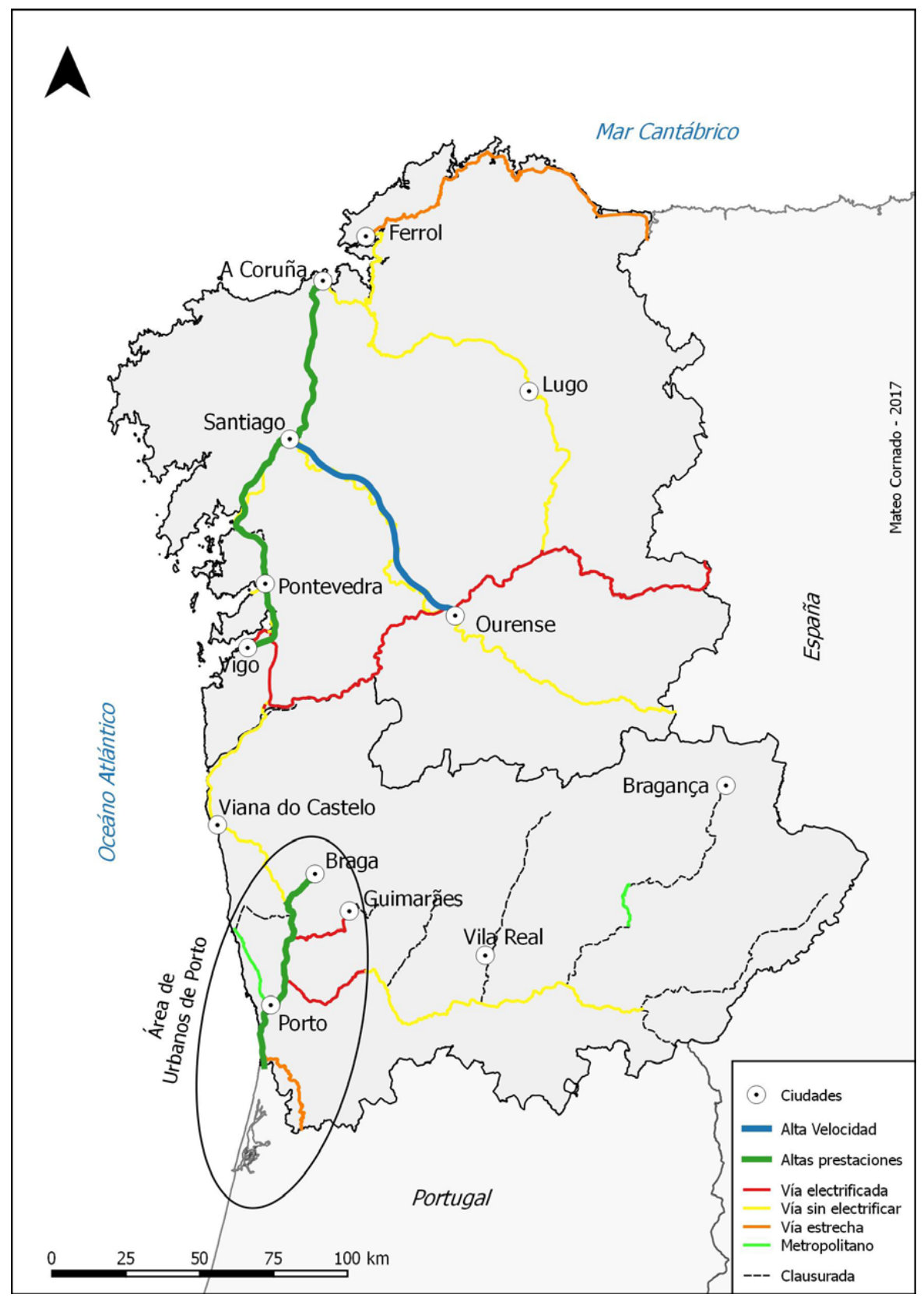

procurar otras alternativas. En la actualidad, la mejora de los servicios entre las diferentes ciudades gallegas, llevó a un aumento considerable del número de usuarios, siendo la línea entre Vigo y A Coruña, una de las principales a nivel estatal, tanto en usuarios como rentabilidad económica (VVAA, 2016). Por otra parte, el establecimiento de un servicio exclusivo para la conexión entre ciudades, no da respuesta a la alta movilidad de las áreas metropolitanas. Lo que supone un grave error en cuanto a la planificación territorial y del sistema de transporte colectivo (Pazos, 2015) (Figura 3).

Una de las principales diferencias entre el Norte de Portugal y Galicia, es el establecimiento de un servicio metropolitano que da respuesta a la alta movilidad en grandes áreas urbanas. Así como en Galicia, tan sólo se puede hablar de esta tipología en un pequeño tramo de $8 \mathrm{~km}$ entre Ferrol y Xubía. En el Norte de Portugal, especialmente en el Gran Porto, existe un sistema metropolitano ferroviario, cuya área de influencia es de $60 \mathrm{~km}$, aglutinando a importantes ciudades como Braga, Guimarães o Aveiro.
Figura 3.Tipología de la red ferroviaria en la Eurorregión en el 2017. Fuente: ADIF 2017 e Infraestruturas de Portugal 2017. 
Esta última, fuera de la propia Región Norte. Tenemos que tener en cuenta que el tren de cercanías o metropolitano, está directamente relacionado con el proceso de metropolitización, ya que permite el desplazamiento diario de miles de trabajadores desde las periferias a los centros de las ciudades, principales generadoras de empleo (Lois, González y Escudero, 2012). Un hecho que si dio en el Gran Porto, donde el ferrocarril tuvo cierto protagonismo en la extensión de la urbanización (Pacheco, 1992). En el año 2015, fueron más de 20 millones los usuarios de los urbanos de Porto (Comboios de Portugal, 2017).

Por el contrario, en Galicia la metropolitización de las principales ciudades comenzó a partir de la década de los 1980s, más tardía que en el Gran Porto, y caracterizada por la universalización del vehículo privado y las mejoras en la accesibilidad con la construcción de diversas autopistas o vías rápidas (Pazos, 2015; Lois e Piñeira, 2011). El predominio de la vivienda unifamiliar en estas dos áreas metropolitanas, establece una gran dependencia del vehículo privado, lo que dificulta el establecimiento de un sistema metropolitano de transporte colectivo. Además, las líneas ferroviarias que atraviesan estas dos áreas metropolitanas apenas cuentan con servicios atractivos para el usuario, número de frecuencias reducido o dificultades para la intermodalidad. Una cuestión disponible en el Gran Porto, donde gracias a la tarjeta Andante, podemos realizar un rápido intercambio de medios de transporte (autobús, metro, tren), mientras que en Galicia, la tarjeta de transporte metropolitano, sólo está disponible para las líneas de autobús urbano y supralocal. También tenemos que tener en cuenta la realidad urbana, no es semejante en ambos espacios. Así como en Galicia, el sistema urbano está caracterizado por su policentrismo (Pazos, 2011), donde ninguna ciudad destaca por encima de las demás y pueda canalizar la mayoría de los desplazamientos, en el Norte de Portugal existe una gran jerarquía en torno a la ciudad de Porto y toda su área metropolitana (Guichard, 1992; López y Pérez, 2015) (Figura 4). De ahí las prioridades de las políticas ferroviarias en los dos espacios sean diferentes (Rodríguez y Pérez, 2007).

\section{Vigo-Porto, única conexión ferroviaria en la Eurorregión}

A pesar de los avances en los últimos años, tanto en Galicia como en el Norte de Portugal, en la dotación y servicios del ferrocarril, lo que supuso un aumento del número de usuarios y una mejor imagen como medio de transporte, el único servicio entre los dos espacios, sigue anclado en el pasado. De hecho, si nos centramos en las características de la red ferroviaria desde A Coruña hasta Lisboa, es decir, gran parte de la fachada atlántica de la Península Ibérica, tan sólo el tramo entre Vigo y la localidad lusa de Nine, no dispone de una vía de altas prestaciones. Una gran diferencia con respecto a la red viaria, donde si existe un eje continuo mediante autopistas, la gallega AP-9 y las lusas A-3 y A-1. Este itinerario se encuentra dentro de la Red Europea de Carreteras, concretamente, la E-01.

El paso fronterizo entre Tui y Valença do Minho mediante el Puente Internacional, inaugurado en 1886, es uno de los 3 que existen entre España y Portugal vía ferrocarril. A pesar de la alta movilidad entre los dos espacios, las características de la infraestructura y sobre todo, del único servicio comercializado conjuntamente por ambas operadoras, provoca que el ferrocarril no sea atractivo para la captación de nuevos usuarios (Palmeiro y Pazos, 2008). En la actualidad, tan sólo existen dos frecuencias por sentido al día, una a primera hora de la mañana y la segunda, en las últimas horas de la jornada laboral. El tiempo del trayecto es de aproximadamente dos horas, al igual que su principal competidor, el autobús. Otra cuestión muy importante son los horarios del servicio ferroviario, ya que están más enfocados al fomento del excursionismo o 


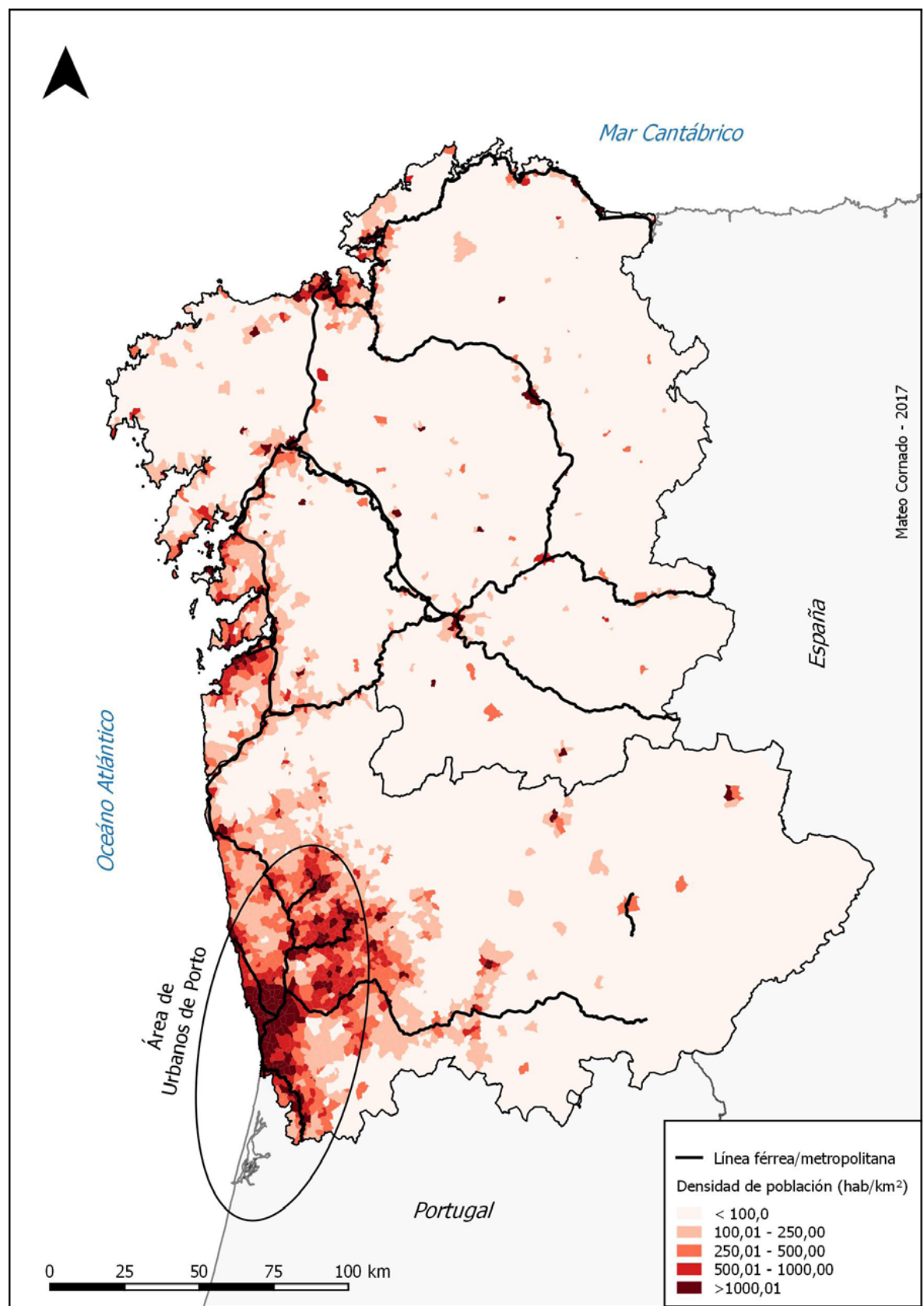

turismo que a la movilidad diaria de personas. Así queda plasmado en los diferentes anuncios de Renfe y CP para la promoción de este enlace internacional. De ahí que una gran parte de los usuarios del Tren Celta sean turistas de Vigo o Porto que deciden visitar los atractivos de la otra ciudad en una jornada.

La mejora de las comunicaciones ferroviarias entre Galicia y el Norte de Portugal, siempre fue una materia presente en las diferentes Cumbres Hispano-Lusas llevadas en los últimos años. Como hemos anunciado anteriormente, se llegaron a presentar varias propuestas para la construcción de una línea de alta velocidad entre Porto y Vigo. Las previsiones establecían un tiempo aproximado de 50 minutos, menos de la mitad de los actuales (VVAA, 2004). Aunque esta posibilidad, supondría un posible aumento de las relaciones entre las dos ciudades, tampoco sería la solución idónea para la vertebración territorial dentro de la Eurorregión, debido a los efectos espaciales del tren de alta velocidad, cómo es el efecto túnel (Gutiérrez, 2004). Entre las diferentes
Figura 4. Densidad demográfica $\left(\mathrm{hab} / \mathrm{km}^{2}\right)$ en la Eurorregión. Año 2011 y red ferroviaria. Fuente: IGE 2017 (Instituto Galego de Estadística) e INE 2017 (Instituto Nacional de Estadística). 
alternativas, existía la posibilidad de conectar la ciudad de Braga de manera directa con Galicia o el aeropuerto de Sa Carneiro (Porto) con la nueva línea ferroviaria. El enlace mediante alta velocidad no solo se llegó a planificar por parte de Portugal. En Galicia, el llamado Eje Atlántico ferroviario, actualmente entre Vigo y A Coruña, se encuentra parcialmente completado. En los primeros proyectos, este eje se trataría de una línea de altas prestaciones entre Ferrol y la frontera portuguesa.

Al mismo tiempo que Portugal y España redactaban y analizaban las diferentes alternativas para la conexión mediante alta velocidad entre los dos estados, la Comisión Europea presentaba en el 2005 la nueva Red Transeuropea de Transporte. En esta nueva estrategia, se describían 30 ejes prioritarios para la mejora de la movilidad y el transporte dentro del continente europeo. De estos 30 proyectos, 2 de ellos, afectaban de lleno a la conexión entre Galicia y el Norte de Portugal. Por una parte, el número 8, Eje Multimodal Portugal/España con el resto de Europa, donde se recoge la mejora del Eje A Coruña-Lisboa-Sines, es decir, la práctica totalidad de la fachada atlántica de la Península Ibérica. El segundo proyecto es el número 19, Interoperabilidad ferroviaria de alta velocidad en la Península Ibérica, con especial atención a la diferencia de ancho de vía de los dos estados con respecto a los demás países de la Unión Europea. También se recogía la mejora de las conexiones mediante el ferrocarril entre Portugal y España, caso del corredor Vigo-Porto (Comisión Europea, 2005). Dentro de la política europea en materia de transporte y movilidad, hay que tener en cuenta además las dos ediciones del Libro Blanco de Transporte, editados en el 2001 y 2011 (Comisión Europea 2001; Comisión Europea 2011). En estos dos documentos, existe una preferencia al fomento del ferrocarril mediante la creación de un único espacio ferroviario europeo, la apertura del mercado ferroviario a la competencia o la mejora de la seguridad. En la actualidad, siguen existiendo diferencias en los sistemas de seguridad, obligando a los maquinistas lusos y gallegos, a cambiar de sistema en el momento que cambian de país. Siendo este uno de los principales problemas a solucionar entre los dos estados. Una de las causas del accidente ferroviario en O Porriño (Galicia) en septiembre del 2016, con 4 fallecidos, fue el uso de diferentes sistemas de seguridad. Para los maquinistas portugueses, de disponer del sistema luso, no habría tenido lugar este accidente.

Suena contradictorio que al mismo tiempo que se llevaban acuerdos entre los dos gobiernos estatales para el establecimiento de las nuevas conexiones mediante velocidad o las directrices por parte de la Unión Europea para la mejora de la movilidad y transporte transfronterizo, se llegase a plantear la posibilidad de la eliminación (2005 y 2011) del único servicio internacional entre Galicia y Portugal. Se puede considerar como un toque de atención a los responsables de ambos países por el teórico abandono del ferrocarril y la pérdida constante de usuarios. Esto provocó que en el 2013, se llevase a cabo una reforma del servicio, originando el actual "Tren Celta". Realmente, lo único que se realizó, fue una eliminación de todas las paradas intermedias y la renovación de los vehículos. La eliminación de las paradas intermedias supuso una mejora considerable de los tiempos, rebajando en más de una hora el necesario para el desplazamiento entre las dos ciudades. Luego, en el 2014, se establecían de nuevo las paradas en Nine, para el enlace con Braga, Viana de Castelo, capital de distrito, y Valença do Minho, sin afectar considerablemente a los tiempos (Figura 5).

Recientemente, el actual gobierno portugués accedió a la mejora de la Linha do Minho. Esto provocará una reducción en los tiempos de desplazamiento y una mejora en la vertebración territorial de la Eurorregión al mejorarse la accesibilidad a los dos espacios. Bien es cierto, que seguirán existiendo ciertas cuestiones a mejorar, para sacar mayor provecho de este medio de transporte. Por ejemplo, la salida sur de Vigo, que permitiría a la nueva estación de Vigo-Urzaiz acceder a los servicios internacionales y a la conexión de los servicios de altas prestaciones hasta A Coruña. En la actualidad, el Tren Celta llega a la estación de Vigo-Guixar, adecuada para las líneas convencionales y 


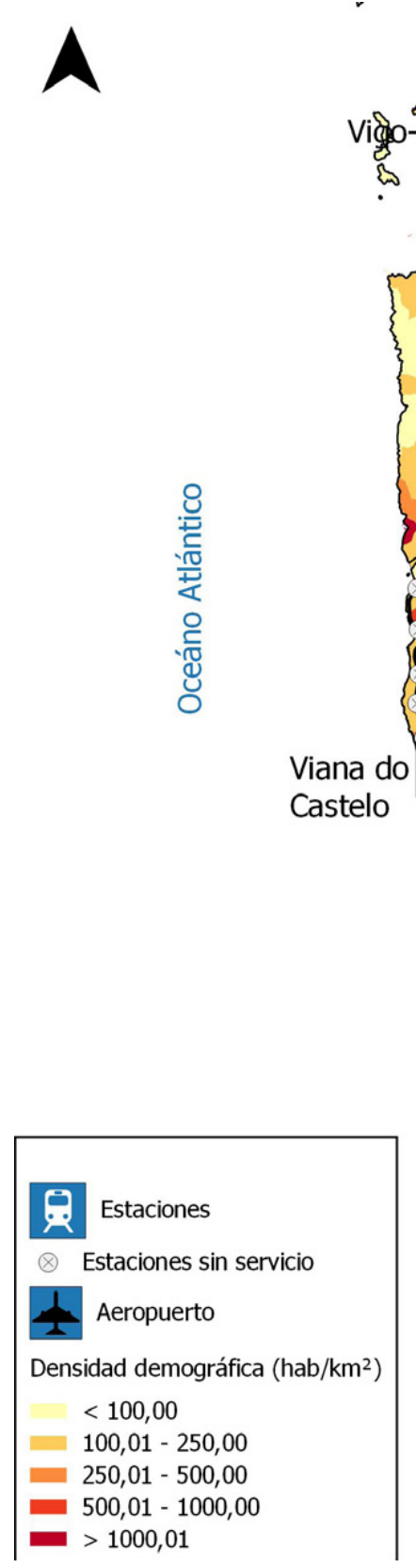

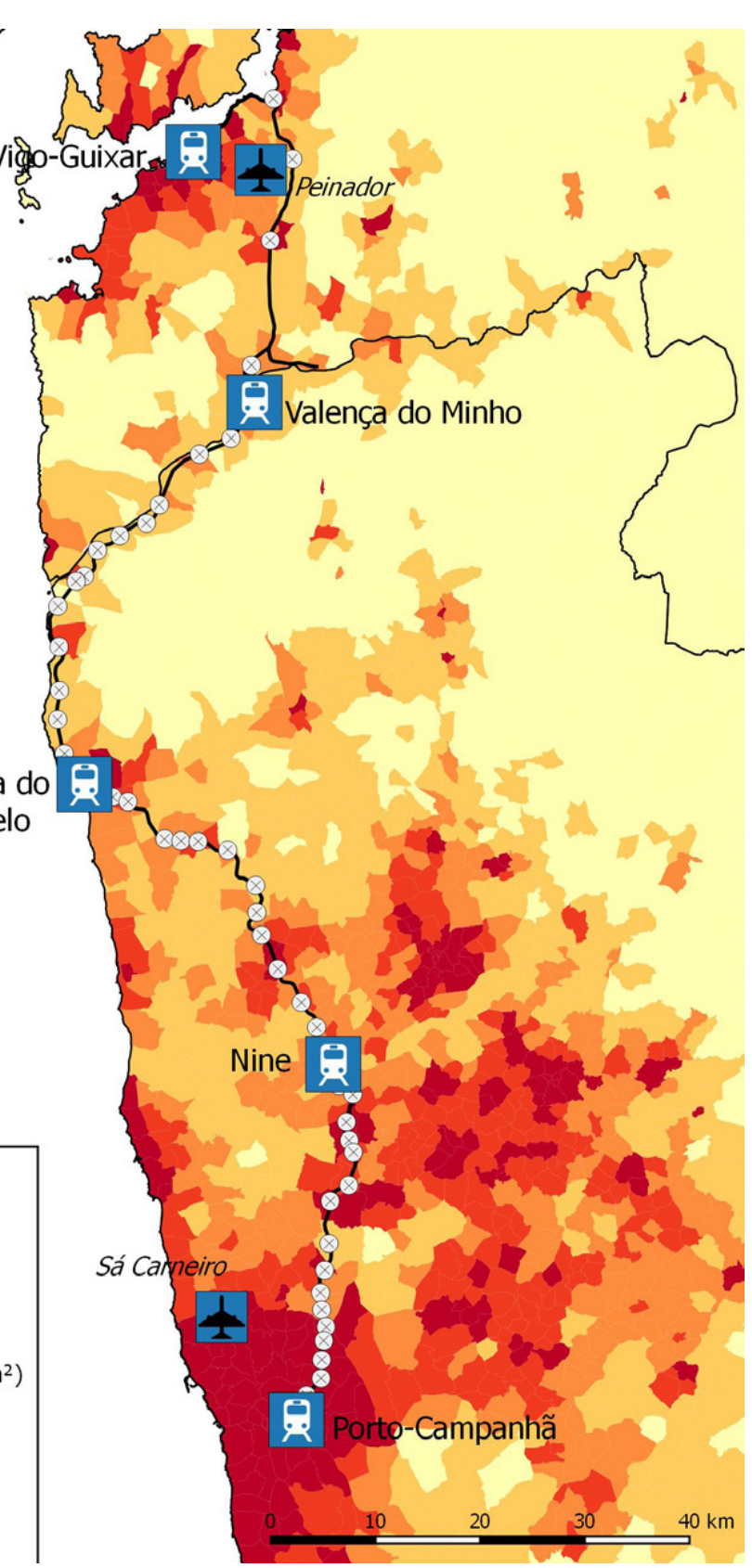

Figura 5. Servicio internacional Porto-Vigo, estaciones con y sin parada, densidad demográfica en el 2011. Fuente: IGE 2017, INE 2017, Renfe 2017 y CP 2017.

los trenes de larga distancia (Barcelona, Madrid y Hendaya), mientras que la de Urzaiz, reinaugurada en 2015, tan sólo dispone de los servicios de altas prestaciones. Por otro lado, también es necesario el establecimiento de una política tarifaría y horaria coordinada entre diferentes medios de transporte a ambos lados de la frontera.

\section{Conclusión}

En un marco actual en el que se procura una mayor integración europea, donde las fronteras políticas ya no son un impedimento para la movilidad de personas y mercancías, es incompresible la falta de una oferta adecuada para la vertebración de ejes y regiones supra-estatales cómo es el caso que estuvimos a analizar. A pesar de todas las directrices marcadas desde la Unión Europea y las fuertes sinergias internas en un 
espacio coma la Eurorregión Galicia-Norte de Portugal, no existe una planificación conjunta en materia de transporte colectivo y en especial, del ferrocarril para este espacio. Actualmente, las ciudadanas gallegas tienen una mayor facilidad para desplazarse hasta Madrid o incluso, otras ciudades de España que a la vecina Portugal. En los últimos años, los tiempos de desplazamiento a la capital española se redujeron drásticamente, competiendo con el vehículo privado y se prevé que en los próximos años, estos lo hagan con el avión.

Bien es cierto que tenemos que tener en cuenta el período en el cual se construye la mayor parte de la red ferroviaria a ambos lados de la frontera, finales del siglo XIX y principios del XX, donde la movilidad trans-fronteriza no tenía la misma magnitud que en la actualidad. De ahí, la falta de conexiones entre Galicia y el Norte de Portugal. Pero por otra parte, desde la entrada de los dos estados en la CEE en 1986, no se llevaron a cabo grandes actuaciones para la mejora de las comunicaciones mediante transporte colectivo. Tan sólo el mantenimiento de un arcaico servicio internacional y puntuales mejoras. Todo lo contrario de lo marcado desde las diferentes directivas europeas y sobre todo, en un momento en el que ferrocarril vuelve a cobrar importancia dentro de la movilidad diaria. No sólo en las áreas metropolitanas, también a media y larga distancia, como se puede comprobar en el caso de Galicia y el resto de España, a pesar de los grandes excesos en su dotación.

Por otra parte, la falta de competencias en materia de infraestructuras y servicios de transporte por parte de la Eurorregión Galicia-Norte de Portugal, dificulta aún más la puesta en marcha de servicios comunes, en un espacio donde conviven 2 políticas diferentes. Incluso podríamos llegar a hablar de 3, en el caso de que la Comunidad Autónoma de Galicia cree su propia operadora ferroviaria. Esta cuestión obliga a diferentes acuerdos entre ambas partes para el establecimiento de servicios comunes, como se pudo ver recientemente con el Tren Celta. En los últimos años, diferentes asociaciones a ambos lados de frontera vienen demandando, no sólo la mejora de la oferta, sino que también la creación de un consorcio de transporte para la Eurorregión, y de esta manera, poder establecer un servicio comercial común que facilite los desplazamientos mediante la integración de tarifas, horarios y la intermodalidad con los transportes urbanos.

La coexistencia de dos sistemas ferroviarios dentro de la propia Eurorregión, no sólo viene derivada de las diferentes políticas ferroviarias llevadas a cabo a lo largo de más de 150 años a ambos lados de la frontera. También hay que tener muy presente las diferencias en la red urbana y la densidad demográfica, claves para comprender la actual situación. Así como en el norte de Portugal, la ciudad de Porto y toda su área metropolitana concentra la mayoría de los desplazamientos, en Galicia, no existe un área urbana de semejantes características, de ahí la falta de una red de cercanías. A pesar de esta diferencia, esto no debe de ser un impedimento para la coordinación y mejora de los servicios entre los dos espacios a analizar. Sobre todo en un momento actual, donde las fronteras ya no son una barrera para la movilidad.

Por último, la nueva decisión del gobierno de Portugal para la mejora de las líneas ferroviarias cara Galicia se convierte en una gran oportunidad en la vertebración y cohesión territorial. Este debe de ser el primer paso para aumentar las relaciones entre los dos espacios de la Eurorregión y dar una respuesta a la alta movilidad mediante el uso de transporte colectivo. 


\section{Q Bibliografía}

» ALBALATE DEL SOL, Daniel y BEL I QUERALT, Germà (2011) Cuando la economía no importa: auge y esplendor de la alta velocidad en España. Revista de Economía Aplicada vol. XIX nº 55, pp. 171-190.

" ALEGRIA, Maria Fernanda (1990) A organização dos transportes em Portugal (1850-1910). As vias e o tráfego. Memórias do Centro de Estudos Geograficos, no 12. Lisboa: Centro de Estudos Geográficos.

» BEL I QUERALT, Germà (2010) España, capital París: origen y apoteosis del Estado radial: del Madrid sede cortesana a la "capital total". Barcelona: Destino.

» BELLET SANFELIU, Carme (2013) Transporte y desarrollo territorial. El estudio de los efectos asociados a la implantación de la alta velocidad ferroviaria a través del caso español. Revista Transporte y Territorio nº 8, pp. 117-137.

" BLANCO CASAL, Alberte (2014) O conflito de intereses no camiño de ferro de Zamora a Ourense. Aproximación histórica ao período 1840-1930. Ourense: Deputación provincial de Ourense.

»CARBALLO LOMBA, Antón (2014) Eurorrexión Galiza-Norte de Portugal: necesidade dunha mobilidade sustentable transfronteiriza. AGALI Journal. Journal of Social Sciences and Humanities, vol. $3 \mathrm{n}^{\circ} 3$.

»COMISIÓN EUROPEA (2001) Libro blanco. La política europea de transportes de cara al 2010: la hora de la verdad. Bruselas. Disponible: https://ec.europa. eu/transport/sites/transport/files/themes/strategies/doc/2001_white_paper/ lb_com_2001_0370_es.pdfConsultado 6/09/2017.

»COMISIÓN EUROPEA (2005) Trans-European transport network. TEN-T priority axes and projects 2005. Bruselas: European Comission, Energy and Transport DG. Disponible: http://ec.europa.eu/ten/transport/projects/doc/2005_ten_t_ en.pdf Consultado 6/09/2017.

» COMISIÓN EUROPEA (2011) Libro blanco del transporte: Hoja de ruta hacia un espacio único europeo de transporte: por una política de transportes competitiva y sostenible. Luxemburgo: Oficina de Publicaciones de la Unión Europea. Disponible: https://ec.europa.eu/transport/sites/transport/files/themes/ strategies/doc/2011_white_paper/white-paper-illustrated-brochure_es.pdf Consultado 6/09/2017.

" DíAZ FERNÁNDEZ, José Antonio (2002) Aproximación a la política del transporte ferroviario en Galicia. Pontevedra: Servicio de Publicacións Deputación de Pontevedra.

" FERNÁNDEZ DÍAZ, Xosé Carlos (2006) El ferrocarril en Galicia: pasado, presente, futuro. Vigo: Ir Indo Edicións.

» FERNÁNDEZ FERNÁNDEZ, Melchor y FERNÁNDEZ GRELA, Manuel (2003) Comparación de estructuras produtivas entre rexións fronterizas: Galicia e a Rexión Norte de Portugal. Revista Galega de Economía vol. 12 nํㅡ, Santiago de Compostela, Universidade de Santiago de Compostela, pp.83-106.

» FLORIDA, Richard; GULDEN, Tim y MELLANDER, Charlotta (2008) The rise of the mega-region. Cambridge Journal of Regions, Economy and Society. 1(3), pp. 459-476.

» GUICHARD, François (1992) Porto, la ville dans sa région: contribution à l'étude 
de l'organisation de l'espace dans le Portugal du nord. París: Fondation Calouste Gulbenkian, Centre Culturel Portugais.

» GUTIÉRREZ PUEBLA, Javier (1998) Redes, espacio y tiempo. Anales de Geografía de la Universidad Complutense no 18 , pp. 65-68.

" GUTIÉRREZ PUEBLA, Javier (2004) El tren de alta velocidad y sus efectos regionales. Investigaciones Regionales, no 5, pp. 199-221.

» LOIS GONZÁLEZ, Rubén Camilo; SANTOS SOLLA, Xosé Manuel; VALCÁRCEL RIVEIRO, Carlos (2002) As relaçons de Portugal com a Ibéria: uma olhada desde a Galiza. Lusotopie no 10, Paris, Ed. Karthala, pp.193-211.

» LOIS GONZÁLEZ, Rubén Camilo y TRILLO SANTAMARÍA, Juan Manuel (2011) La frontera como motivo de atracción: una breve mirada a las relaciones Galicia-Região Norte. Geopolítica vol.2 no 1, pp.109-134.

» LOIS GONZÁLEZ, Rubén Camilo y PIÑEIRA MANTIÑÁN María José (2011) A rede urbana e a rápida urbanización do territorio. En: Piñeira Mantiñán Mํㅡ. J. y Santos Solla X.M. (Coords.). Xeografía de Galicia. Vigo, Ed. Xerais. pp. 159-227

" LOIS GONZÁLEZ, Rubén Camilo; GONZÁLEZ PÉREZ, Jesús Manuel y ESCUDERO GÓMEZ, Luis Alfonso (2012) Los espacios urbanos. El estudio geográfico de la ciudad y la urbanización. Madrid: Biblioteca Nueva.

» LÓPEZ CARRO, Manuel Ramiro y PÉREZ GONZÁLEZ, Arturo (2015) Lectura comparativa das xeografías urbanas de Vigo e do Porto. Agali Journal. Journal of Social Sciences and Humanities vol. 4 ํㅜ 4 .

» NÁRDIZ ORTIZ, Carlos (1996) Desarrollo histórico de la red ferroviaria del noroeste de España. En: Rodríguez Bugarín, M. y Nárdiz Ortiz, C. (Coords.) El ferrocarril en el noroeste de España. A Coruña, Universidade de A Coruña. pp.57-79.

» PACHECO, Elsa Maria (1992) Os transportes colectivos rodoviários no Grande Porto. Revista da Facultade de Letras-Geografia 1 (8) pp. 5-64.

»PACHECO, Elsa Maria (2001) Alteração das Acessibilidades e Dinâmicas Territoriais na Região Norte: expectativas, intervenções e resultantes. Edição electrónica da Tese de Doutoramento, GEDES/ Faculdade de Letras da Universidade do Porto.

» PALMEIRO PIÑEIRO, Xosé Luis y PAZOS Otón, Miguel (2008) La Eurorregión Galicia-norte de Portugal: una aproximación a la movilidad en el contexto ibérico. Estudios Geográficos vol. 69 n²64, pp. 215-245.

» PALMEIRO PIÑEIRO, Xosé Luis (2011) Galicia no contexto internacional. En: Piñeira Mantiñán Ma J. y Santos Solla X.M. (Coords.). Xeografía de Galicia. Vigo, Ed. Xerais, pp. 429-467.

» PAZOS OTÓN, Miguel (2003) Movilidad de la población en la Galicia Occidental. El Eje Urbano Atlántico gallego. Tesis doctoral en CD-ROM. Santiago de Compostela, Universidade de Santiago de Compostela.

»PAZOSOTÓN, Miguel (2011) As infraestruturas de transporte e a desequilibrada vertebración de Galicia. En: Piñeira Mantiñán Mํㅣ. y Santos Solla X.M. (Coords.). Xeografía de Galicia. Vigo, Ed. Xerais, pp. 133-154.

» PAZOS OTÓN, Miguel (2015) Mobilidade e sistema urbano en Galicia. En: Lois González, R.C. y Pino Vicente, D. (Coords.). A Galicia urbana. Vigo, Ed. Xerais, pp. 79-99.

»PAZOS OTÓN, Miguel; VARELA CORNADO, Mateo y LOIS GONZÁLEZ, Rubén Camilo (2015) O corredor urbano atlántico de Galicia. Cara unha nova xeografía da mobilidade?. GOT no 7 (junho), Porto, Centro de Estudos de Geografia e 
Ordenamento do território, pp. 283-300, dx.doi.org/10.17127/got/2015.7.013

» RAMOS MELERO, Rodolfo (2002) El futuro de la política ferroviaria en España. Boletín Económico de ICE n- 2734 del 1 al 7 de junio de 2002, pp. 9-21.

» RODRÍGUEZ BUGARÍN, Miguel y PÉREZ BABO, António (Coords.) (2007) A rede ferroviária interior na Euro-regiao Galiza-Norte de Portugal. Porto: Eixo Atlántico do Noroeste Peninsular.

" TROIN, Jean François (1995) Rail et aménagement du territoire. Des héritages aux noveaux défis. Aix-en-Provence: Edisud.

» URRY, John (1999) Mobile Cultures. Departament of Sociology, Lancaster University. Lancaster.

" VVAA (2004) Estudio de viabilidad técnica, económica y medioambiental de la conexión Hispano-Lusa en Alta Velocidad Vigo-Oporto. Demanda actual y prognosis.

»Disponible: http://www.infraestruturasdeportugal.pt/sites/default/files/ altavelocidade_estudoprocura_porto_vigo_2004.pdf Consultado 28/08/2017

»VVAA (2016) Observatorio del ferrocarril en España. Informe 2015. Fundación de los Ferrocarriles. Disponible: https://www.fomento.gob.es/NR/rdonlyres/2D6FF36618BE-4D71-9CoB-DFA456575AFB/142179/Informe_OFE2015.pdf Consultado 7/09/2017

"VVAA (2017) Observatorio transfronterizo España-Portugal (OTEP). Documento no 8, mayo 2017, Secretaría General de Transporte; Ministerio de Fomento,

》Disponible:https://www.fomento.gob.es/NR/rdonlyres/8D19946C-814B-468DBBE5 07BAA364DC81/143436/Informe_OTEP8.pdf Consultado 7/09/2017

»Fuentes de internet:

» ADIF, Administrador de Infraestructuras Ferroviarias, (2017) <www.adif.es/gl_ ES/index.shtml> Consultado 28/08/2017

»Comboios de Portugal, (2017) <www.cp.pt> Consultado 30/08/2017

» IGE, Instituto Galego de Estadística, (2017) <www.ige.eu> Consultado 5/09/2017

»Infraestruturas de Portugal, (2017) <www.infraestruturasdeportugal.pt> Consultado 28/08/2017

»INE, Instituto Nacional de Estatística, (2017) <www.ine.pt> Consultado 5/09/2017

» RENFE, Red Nacional de los Ferrocarriles Españoles, (2017) <www.renfe.es> Consultado 30/08/2017

\section{Mateo Varela Cornado / mateo.cornado@gmail.com}

Graduado en Geografía y ordenación del territorio en la Universidad de Santiago de Compostela. Posteriormente, posgraduado en Planificación y Gestión del desarrollo territorial en la misma universidad. Actualmente, estudiante de doctorado en las Universidades de Santiago de Compostela (España) y Toulouse-II Jean Jaurès (Francia). Sus líneas de investigación son la geografía de los transportes y el turismo. 